\title{
High-Throughput Metabolomics for Discovering Potential Biomarkers and Identifying Metabolic Mechanisms in Aging and Alzheimer's Disease
}

OPEN ACCESS

Edited by:

Lei Deng,

Central South University, China

Reviewed by:

Jihui Lyu,

Beijing Geriatric Hospital, China

Xiaojuan Han,

Shandong Provincial Hospital, China

*Correspondence:

Fan Wang

yifan.701@163.com

Maoqing Wang

wang_maoqing@126.com

†These authors have contributed equally to this work

Specialty section:

This article was submitted to

Molecular Medicine,

a section of the journal

Frontiers in Cell and Developmental

Biology

Received: 04 September 2020

Accepted: 04 February 2021

Published: 25 February 2021

Citation:

Xie K, Qin Q, Long Z, Yang Y,

Peng C, Xi C, Li L, Wu Z, Daria V,

Zhao $Y$, Wang F and Wang $M$ (2021)

High-Throughput Metabolomics

for Discovering Potential Biomarkers and Identifying Metabolic

Mechanisms in Aging and Alzheimer's

Disease.

Front. Cell Dev. Biol. 9:602887. doi: 10.3389/fcell.2021.602887

\begin{abstract}
Kun Xie ${ }^{1 \dagger}$, Qi Qin't, Zhiping Long1, Yihui Yang1, Chenghai Peng ${ }^{3}$, Chunyang Xi4, Liangliang $\mathrm{Li}^{1}$, Zhen Wu ${ }^{1}$, Volontovich Daria', Yashuang Zhao' ${ }^{1}$, Fan Wang ${ }^{1 *}$ and Maoqing Wang ${ }^{5 *}$
\end{abstract}

\begin{abstract}
1 Department of Epidemiology, School of Public Health, Harbin Medical University, Harbin, China, ${ }^{2}$ Department of Neurology, Innovation Center for Neurological Disorders, National Clinical Research Center for Geriatric Diseases, Xuan Wu Hospital, Capital Medical University, Beijing, China, ${ }^{3}$ The Forth Affiliated Hospital of Harbin Medical University, Harbin, China, ${ }^{4}$ Department of Orthopedic Surgery, The Second Affiliated Hospital of Harbin Medical University, Harbin, China, ${ }^{5}$ National Key Disciplines of Nutrition and Food Hygiene, Department of Nutrition and Food Hygiene, School of Public Health, Harbin Medical University, Harbin, China
\end{abstract}

Alzheimer's disease (AD) is an aging-related neurodegenerative disease. We aimed to investigate the metabolic mechanisms of aging and $A D$ and to identify potential biomarkers for the early screening of $A D$ in a natural aging population. To analyze the plasma metabolites related to aging, we conducted an untargeted metabolomics analysis using ultra-high-performance liquid chromatography/quadrupole time-of-flight mass spectrometry in a two-stage cross-sectional study. Spearman's correlation analysis and random forest were applied to model the relationship between age and each metabolite. Moreover, a systematic review of metabolomics studies of AD in the PubMed, Cochrane and Embase databases were searched to extract the differential metabolites and altered pathways from original studies. Pathway enrichment analysis was conducted using Mummichog. In total, 669 metabolites were significantly altered with aging, and 12 pathways were enriched and correlated with aging. Three pathways (purine metabolism, arginine and proline metabolism, and the TCA cycle) were shared between aging and AD. Arginine and proline metabolism play a key role in the progression from healthy to mild cognitive impairment and to $A D$ in the natural aging population. Three metabolites, 16-a-hydroxypregnenolone, stearic acid and PC[16:0/22:5(4Z,7Z,10Z,13Z,16Z)] were finally proposed as potential markers of $A D$ in the natural aging population. The underlying mechanism shared between aging and $A D$ and the potential biomarkers for $A D$ diagnosis were proposed based on multistep comparative analysis.

Keywords: aging, Alzheimer's disease, mild cognitive impairment, metabolic biomarkers, metabolomics

\footnotetext{
Abbreviations: Ach, acetylcholine; $\mathrm{AD}$, Alzheimer's disease; $\mathrm{A} \beta$, amyloid beta; $\mathrm{BDNF}$, brain-derived neurotrophic factor; CNS, central nervous system; CDP-choline, cytidine diphosphate choline; DHA, Docosahexaenoic acid; ESI, electrospray ionization; $\mathrm{CN}$, healthy controls; MS, mass spectrometry; MCI, mild cognitive impairment; NMR, nuclear magnetic resonance; NO, nitric oxide; QUADAS, Quality Assessment of Diagnostic Accuracy Studies; QC, quality-control; RSD, relative standard deviations.
} 


\section{INTRODUCTION}

Alzheimer's disease (AD) is an aging-related neurodegenerative disease characterized by senile plaques caused by amyloid beta $(\mathrm{A} \beta)$ and neurofibrillary tangles containing hyperphosphorylated tau-protein. Current estimates suggest that 44 million people live with dementia worldwide. This figure is predicted to more than triple by 2050 as the population ages; at this time, the annual cost of dementia in the United States alone may exceed US\$600 billion (Lane et al., 2017; Nichols et al., 2019). Mild cognitive impairment (MCI, "amnestic MCI" is seen as a prodromal stage of Alzheimer's disease) represents the clinically diagnosed predementia stage. Several studies have shown that the pathological changes of $\mathrm{AD}$ begin several years before the onset of evident memory impairment (Jack et al., 2010; Fleisher et al., 2012). However, the current diagnosis is based on clinical symptoms of $\mathrm{AD}$ combined with pathological alterations, such as a decrease of cerebrospinal fluid $\mathrm{A} \beta 42$ or an increase in $\mathrm{p}$-tau or $\mathrm{t}$-tau protein, which may not identify a substantial number of asymptomatic individuals who will develop $\mathrm{AD}$ later. Thus, it is urgent to investigate the physiology of $\mathrm{AD}$ and/or MCI and detect early biomarkers to improve the quality of life of those affected by this disease.

Although genetic factors involved in the development of $\mathrm{AD}$ have been identified, studies have also suggested that aging is the major risk factor. After the age of 60 years, the incidence of $\mathrm{AD}$ was found to double with every 5 years increase in age (Mayeux and Stern, 2012). Although the mechanisms triggering alterations associated with both aging and $\mathrm{AD}$ are not completely understood, they have been simultaneously divided into at least two aspects: oxidative stress and inflammation. First, increasing oxidative stress is observed in the contexts of aging, $\mathrm{AD}$ and/or MCI in terms of membrane lipids (Mielke and Lyketsos, 2006; Zhu et al., 2006; Markesbery and Lovell, 2007), proteins (Poon et al., 2006; Greilberger et al., 2008), and mitochondrial DNA (Bender et al., 2006; Wang et al., 2006). Second, inflammation is found to be a considerable driving force of aging and $\mathrm{AD}$. In addition, recent studies have identified common histological changes (Ohm et al., 1995; Naslund et al., 2000) and modulation of neurotransmission (Kern and Behl, 2009), including hypofunction of the cholinergic system, in $\mathrm{AD}$ and aging (Bartus, 2000; Gron et al., 2006). Thus, the relationship between aging and $\mathrm{AD}$ can be revealed.

Metabolomics has been widely used to provide an overall description of metabolic profiles in pathological or physiological processes (Psychogios et al., 2011). Metabolomics offers quantitative measurement of final products downstream of interactions among genes, proteins and various influences. Compared with genomics and proteomics, metabolomics is regarded as an optimal platform to describe a dynamic physiological process and integral disease response. Evidence has shown that changes in metabolites are significant early indicators of diseases (Nicholson et al., 2008). A growing body of literature has already reported distinct perturbed sets of metabolites in the contexts of aging and AD (Yu et al., 2012; Hertel et al., 2016; Rist et al., 2017; Wilkins and Trushina, 2017). Thus, metabolomics is also a promising tool to systematically assess changes of small molecules in both natural aging and $\mathrm{AD}$ populations and provides clues essential for the early diagnosis of AD. However, little effort to date has been made to detect and compare the metabolites and metabolic pathways between $\mathrm{AD}$ and natural aging.

Therefore, we carried out a metabolomics analysis of agingrelated metabolites and a systematic review of differential metabolites related to $\mathrm{MCI}$ and $\mathrm{AD}$. Based on these findings, we aimed to explore similar metabolomic signatures among $\mathrm{AD}$, MCI and aging, which could help explain the high incidence of $\mathrm{AD}$ in older populations and suggest novel markers to identify the earliest phase of $\mathrm{AD}$ in the natural aging population.

\section{MATERIALS AND METHODS}

\section{Global Plasma Metabolic Profiling Analysis of Aging in a Two-Stage Cross-Sectional Study Study Sample Collection and Detection}

Subjects were enrolled from community-dwelling individuals from the Xiangfang community and surrounding villages in the city of Harbin and orthopedic and ophthalmic patients attending the Second Affiliated Hospital of Harbin Medical University. The training set consisted of 119 participants enrolled in the first half year of 2010, while the testing set of 64 individuals was enrolled in the next half year. All subjects completed a comprehensive questionnaire to obtain information about sociodemographic characteristics, lifestyle, and history of some diseases, such as diabetes, hypertension and heart disease. Fasting peripheral venous blood (5 ml) was collected using an EDTA tube and treated with centrifugation for $10 \mathrm{~min}$ at $3,000 \mathrm{rpm}$ and $4^{\circ} \mathrm{C}$. Then, samples were frozen at $-80^{\circ} \mathrm{C}$ prior to measurement.

We carried out this study after obtaining written informed consent from all the study subjects and approval from the Human Research and Ethics Committee of Harbin Medical University. All experiments, including relevant details, were performed in accordance with relevant guidelines and regulations.

A detailed description of the experimental protocol of metabolic profiling analysis for plasma by UPLC/Q-TOF-MS/MS and the method of metabolites identification were provided in Supplementary Materials 1.1.

\section{Statistical Analysis}

The metabolites detected in both the training and testing sets were considered the stably detected metabolites. Correlations between ion intensities of metabolites and age were calculated by Spearman's correlation analysis. The analytes with $P$-values less than 0.05 were selected for the following statistical analyses. The set of aging-related metabolites was chosen according to the importance score given by random forest, in which age served as the dependent variable and all Spearmancorrelated features served as independent variables. A total of 30 variables were assigned variable importance scores and identified with the HMDB database. All the Spearman-correlated metabolites were analyzed for pathway enrichment analysis 
using Mummichog ${ }^{1}$. All calculations were performed with the R statistical platform, version 3.4.4.

\section{Systematic Review of Metabolomics Studies of $A D$ and $\mathrm{MCl}$ \\ Literature Search Strategy}

We conducted an English language literature search for metabolomic studies of MCI and AD. The search was conducted in PubMed, Cochrane and Embase through August 27, 2018 with the search terms "Alzheimer disease," "Alzheimer's disease," and "mild cognitive impairment" combined with "metabolomics," "metabonomics," "GC-MS," and "LC-MS." Both automatic retrieval and manual retrieval were used for the literature search. In addition, we augmented the search by a snowball strategy, screening the references of original texts and reviews.

A detailed description for the criteria of literation inclusion and exclusion and data extraction was provided in Supplementary Materials 1.2.

\section{Quality Assessment of Individual Studies}

The QUADAS (Lumbreras et al., 2008) (Quality Assessment of Diagnostic Accuracy Studies) is a well-established tool used for the appraisal of quality issues of omics-based studies investigating new diagnostic tests. QUADOMICS included sixteen questions, where the risk of bias could be appraised with respect to four aspects: "patient selection," "index test," "reference standard," and "flow and timing" (Whiting et al., 2011). Possible answers for each item were Y (criteria achieved), N (criteria not achieved), ? (unclear), and NA (not applicable).

\section{Pathway Enrichment Analysis}

We directly extracted the enriched pathways from original studies. Moreover, we conducted pathway enrichment analyses based on all the metabolites extracted from original studies using online analysis platform MetaboAnalyst ${ }^{2}$. All these pathways were combined and categorized according to different biosamples (blood, urine, tissue, or CFS) or comparisons.

\section{RESULTS}

\section{Global Plasma Metabolic Profiling Analysis of Aging in a Two-Stage Cross-Sectional Study}

\section{Demographical Characteristics of the Study} Population

The training set involved 119 participants, aged 32-82 years, with an average age of 58.66 years and BMI of $24.10 \mathrm{~kg}$ $\mathrm{m}^{-2}$. The testing set contained 64 people, aged from 25 to 85 years, with a mean age of 58.98 years and BMI of $23.93 \mathrm{~kg} \mathrm{~m}^{-2}$. Other information on education, smoking, alcohol consumption, chronic diseases and exercises is shown in Supplementary Table 1. No significant difference of any characteristics was observed.

${ }^{1}$ http://mummichog.org/

${ }^{2} \mathrm{https://www.metaboanalyst.ca/}$

\section{Quality Assessment of the Metabolomics Platform}

Quality control (QC) samples were all clustered tightly in the twostage cross-sectional detection. The relative standard deviations (RSDs;\%) of the retention time and peak area ranged from 0 to 0.73 and 0.8 to 4 , respectively, in the intra-batch assay and ranged from 0.1 to 2.7 and 1.5 to 6.2 , respectively, in the inter-batch assay. The results showed that the stability of the UPC/Q-TOF MSMS platform was excellent throughout the run and was sufficient to ensure data quality for further global metabolic profiling analyses.

\section{Identification of Aging-Related Metabolites and Pathway Enrichment Analysis}

By including all duplicated metabolites in Spearman correlation analysis, we found that the ion intensities of 381 metabolites in $\mathrm{ESI}^{-}$and 288 metabolites in $\mathrm{ESI}^{+}$were significantly associated with age $\left(P<0.05\right.$, Excel $\left.1 \& 2^{3}\right)$. Then, we employed an additional statistical method, random forest, to screen out the top 30 metabolites among all 669 metabolites, and 26 of them were identified successfully (Table 1 and Supplementary Figure 1). Several classes of metabolites were observed twice or more often, such as dipeptides, long-chain fatty acids, triterpenoids, steroid glucuronide conjugates, fatty acid esters and phosphatidylcholines.

Table 2 summarizes the pathways enriched by all metabolites correlated with aging using Mummichog. A total of 12 pathways were significantly perturbed with a $P<0.05$.

\section{Systematic Review of Metabolomics Studies of $A D$ and $\mathrm{MCl}$ \\ Literature Retrieval}

In total, 494 articles from PubMed, 23 articles from Cochrane, and 64 articles from Embase were retrieved from automatic electronic searches. A total of 581 publications were examined through title and abstract screening. There were 113 records left after excluding the literatures that were duplicates, reviews, or not related to the research topic (e.g., mechanisms or drug use exploration and technology assessment). Additionally, 9 articles were manually retrieved after searching references from original articles. After careful full-text screening, 67 studies were finally included in this systematic review (Supplementary Figure 2).

\section{Description of Included Studies}

Supplementary Table 2 summarizes the characteristics of the 67 included studies regarding the number of subjects, sample source, sample type, platforms and outcomes. Of the 67 studies, the number of $\mathrm{AD}$ cases ranged from 7 to 1,356 , the number of MCI cases ranged from 10 to 356 , and the number of healthy controls ranged from 7 to 23,882 . The detection platform includes LC-MS in 28 studies, GC-MS in 13 studies, multiple platforms in 11 studies, nuclear magnetic resonance (NMR) in 4 studies, and other platforms in 8 studies. Various biosamples used included serum (18 studies), plasma (16 studies), brain tissue (10 studies), CSF (8 studies), CSF and plasma (5 studies), brain tissue and plasma ( 1 study), urine (4 study), and others ( 5 studies). Additionally, $83.58 \%$ of the studies were case-control

${ }^{3}$ https://doi.org/10.6084/m9.figshare.13621664 
TABLE 1 | Top 30 aging-related metabolites in plasma screened by random forest.

\begin{tabular}{|c|c|c|c|c|c|c|}
\hline Categories/Number & $\mathbf{R T}^{\mathrm{a}}$ & Mass & HMDB ID & Metabolites' name & Additive ion & ESI mode \\
\hline 1 & 2.81 & 283.0861 & HMDB0028853 & Glycyl-Tyrosine & $\mathrm{M}+\mathrm{FA}-\mathrm{H}$ & ESI- \\
\hline 3 & 2.67 & 265.1484 & HMDB0029008 & Phenylalanyl-Valine & $\mathrm{M}+\mathrm{H}$ & ESI+ \\
\hline \multicolumn{7}{|c|}{ Long-chain fatty acids } \\
\hline 4 & 3.71 & 307.1821 & HMDB0000672 & Hexadecanedioic acid & $\mathrm{M}+\mathrm{Na}-2 \mathrm{H}$ & ESI- \\
\hline \multicolumn{7}{|l|}{ Triterpenoids } \\
\hline 7 & 4.49 & 449.2562 & HMDB0002385 & Celastrol & $\mathrm{M}-\mathrm{H}$ & ESI- \\
\hline 8 & 3.94 & 551.3198 & HMDB0004309 & Triterpenoid & $\mathrm{M}-\mathrm{H}$ & ESI- \\
\hline \multicolumn{7}{|c|}{ Steroid glucuronide conjugates } \\
\hline 9 & 3.61 & 597.3527 & HMDB0002513 & Lithocholate 3-O-glucuronide & $\mathrm{M}+\mathrm{FA}-\mathrm{H}$ & ESI- \\
\hline \multicolumn{7}{|l|}{ Phosphatidylcholine } \\
\hline 13 & 9.16 & 758.5624 & HMDB0007880 & $P C[14: 0 / 20: 2(11 Z, 14 Z)]$ & $\mathrm{M}+\mathrm{H}$ & ESI+ \\
\hline 14 & 9.42 & 846.5454 & нМDв0007989 & $\mathrm{PC}[16: 0 / 22: 5(4 Z, 7 Z, 10 Z, 13 Z, 16 Z)]$ & $M+K$ & ESI + \\
\hline \multicolumn{7}{|c|}{ 1,2-diacylglycerol-3-phosphates } \\
\hline 15 & 9.41 & 701.5504 & HMDB0114824 & 1,2-diacylglycerol-3-phosphates & $\mathrm{ESI}+$ & \\
\hline \multicolumn{7}{|c|}{ 1-acylglycerol-3-phosphates } \\
\hline 16 & 4.19 & 485.2756 & HMDB0114752 & LysoPA[22:4(7Z,10Z,13Z,16Z)/0:0] & $\mathrm{M}-\mathrm{H}$ & ESI- \\
\hline \multicolumn{7}{|c|}{ Gluco/mineralocorticoids, progestogins and derivatives } \\
\hline 17 & 3.71 & 369.1899 & HMDB0000315 & 16-a-Hydroxypregnenolone & $M+K-2 H$ & ESI- \\
\hline \multicolumn{7}{|c|}{ Phenylpropanoic acids } \\
\hline 18 & 2.59 & 145.0582 & HMDB0001955 & 3-Phenylbutyric acid & $\mathrm{M}-\mathrm{H} 2 \mathrm{O}-\mathrm{H}$ & ESI- \\
\hline \multirow[t]{2}{*}{22} & 4.00 & 735.3353 & HMDB0033168 & (15a,20R)-Dihydroxypregn-4-en-3-one & $\mathrm{M}+\mathrm{K}-2 \mathrm{H}$ & ESI- \\
\hline & & & & 20-[glucosyl-(1- > 4)-6-acetyl-glucoside] & & \\
\hline \multicolumn{7}{|c|}{ Medium-chain fatty acids } \\
\hline 23 & 3.55 & 167.1402 & HMDB0000947 & Undecanoic acid & $\mathrm{M}-\mathrm{H} 2 \mathrm{O}-\mathrm{H}$ & ESI- \\
\hline \multicolumn{7}{|l|}{ Oligopeptides } \\
\hline 24 & 3.83 & 444.273 & HMDB0012936 & Dynorphin B (10-13) & $\mathrm{M}-\mathrm{H}$ & ESI- \\
\hline \multicolumn{7}{|c|}{ Purine $2^{\prime}$-deoxyribonucleosides } \\
\hline 25 & 2.66 & 275.065 & HMDB0000071 & Deoxyinosine & $\mathrm{M}+\mathrm{Na}$ & ESI+ \\
\hline \multicolumn{7}{|c|}{ Prostaglandins and related compounds } \\
\hline 26 & 3.73 & 315.189 & HMDB0060046 & 15d PGD2 & $\mathrm{M}-\mathrm{H} 2 \mathrm{O}-\mathrm{H}$ & ESI- \\
\hline
\end{tabular}

${ }^{a}$ Rention time. ${ }^{b}$ The HMDB identifier of the metabolite. Bold, 3 metabolites related to both aging and AD, including 16-a-Hydroxypregnenolone, stearic acid, and $P C[16: 0 / 22: 5(4 Z, 7 Z, 10 Z, 13 Z, 16 Z)]$.

studies, and $41.79 \%$ of the subjects in these studies were European (Supplementary Figure 3).

\section{Quality Assessment of Eligible Studies}

According to the QUADOMICS tool, 30 out of 67 articles were unable to avoid overfitting due to the lack of a statistical approach such as cross-validation or an independent test set. All of the articles explored differences in biomarkers between overt cases and healthy individuals and could be categorized into preliminary phase 1 studies. Items with respect to the representative feature of the included subjects and the availability of the clinical data were not applicable for all articles. Supplementary Table 3 presents a detailed assessment for all 67 studies. 
TABLE 2 | Aging-related pathways enriched by spearman-correlated metabolites.

\begin{tabular}{|c|c|c|c|}
\hline Pathways' name & Total no. & Hits no. & $P$-value \\
\hline Carnitine shuttle $e^{a}$ & 15 & 4 & 0.021 \\
\hline Omega-3 fatty acid metabolism ${ }^{a}$ & 5 & 2 & 0.035 \\
\hline $\begin{array}{l}\text { Ascorbate (Vitamin C) and Aldarate } \\
\text { Metabolismb }\end{array}$ & 2 & 2 & 0.007 \\
\hline Glutamate metabolism ${ }^{\mathrm{b}}$ & 6 & 3 & 0.010 \\
\hline Biopterin metabolism & 10 & 4 & 0.012 \\
\hline TCA cycle ${ }^{\mathrm{b}}$ & 3 & 2 & 0.019 \\
\hline $\begin{array}{l}\text { Vitamin B3 (nicotinate and } \\
\text { nicotinamide) metabolismb }\end{array}$ & 15 & 5 & 0.021 \\
\hline Purine metabolism ${ }^{b}$ & 23 & 7 & 0.023 \\
\hline Tyrosine metabolism ${ }^{\mathrm{b}}$ & 31 & 9 & 0.026 \\
\hline Aspartate and asparagine metabolism ${ }^{b}$ & 31 & 9 & 0.026 \\
\hline Carnitine shuttle $^{b}$ & 12 & 4 & 0.031 \\
\hline Leukotriene metabolism ${ }^{\mathrm{b}}$ & 21 & 6 & 0.044 \\
\hline Arginine and Proline Metabolism ${ }^{b}$ & 17 & 5 & 0.045 \\
\hline
\end{tabular}

\section{Metabolites Related to the Occurrence and Progression of $A D$ and $\mathrm{MCl}$}

Supplementary Tables 4-8 presents the metabolites that were extracted as biological markers in original metabolomics studies. There were 830 altered analytes in the comparison of $\mathrm{AD}$ VS. CN, with 137 analytes reported twice or more often (Supplementary Table 4). Tryptophan was the most commonly detected metabolite, followed by palmitic acid, arginine, and L-phenylalanine. The abundance of 293 metabolites was significantly altered in the comparison of MCI VS. CN (Supplementary Table 5); tryptophan was detected 3 times, and 7 other metabolites, such as 5-hydroxytryptophan, L-phenylalanine and L-arginine, were identified twice. For AD VS. MCI, a total of 120 metabolites were affected, including tryptophan (reported 2 times) and histidine (Nichols et al., 2019; Supplementary Table 6). In the prospective studies, 9 and 26 metabolites were found to be related to the progression from $\mathrm{CN}$ to $\mathrm{AD}$ (CN_AD) and MCI to AD (MCI_AD), respectively (Supplementary Tables 7, 8).

Table 3 illustrates the duplicate metabolites between $\mathrm{AD}$ VS. CN and CN_AD VS. CN. Six metabolites (palmitic acid, stearic acid, linoleic acid, glutamine, oleic acid, and myristic acid) were detected in both retrospective case-control and prospective nested case-control studies, and all of these metabolites were detected in serum. In particular, 5 (all except myristic acid) of these 6 metabolites have been found in brain tissue. Additionally, three metabolites (arginine, creatine, and histidine) were detected in both AD VS. MCI and MCI_AD VS. MCI (Table 3), and two (arginine and histidine) of them were confirmed in CSF.

\section{Metabolic Pathways Associated With $A D$ and $\mathrm{MCI}$}

As shown in Supplementary Table 9, there are 53 pathways that exhibited significant alterations in the comparison of $\mathrm{AD}$ VS. CN. These pathways were mainly related to amino acid metabolism (including alanine, aspartate and glutamate metabolism and arginine and proline metabolism), tryptophan metabolism, the TCA cycle, and purine metabolism. Additional analysis for the comparison of MCI VS. CN showed 39 metabolic pathways associated with MCI, including lysine metabolism, tryptophan metabolism, polyamine metabolism, and the urea cycle. Comparison of AD VS. MCI presented 38 altered pathways.

As shown in Figure 1, a total of 15 pathways were shared in the comparison between AD VS. CN and MCI VS. CN, which indicated that the metabolic mechanisms of $\mathrm{AD}$ and MCI share similar pathological alterations. Moreover, four pathways were shared between AD VS. MCI and MCI_AD VS. MCI, including lysine metabolism, polyamine metabolism, catecholamine metabolism, and prostaglandin 2 biosynthesis and metabolism. These pathway alterations indicated pathological progression from MCI to $\mathrm{AD}$.

TABLE 3 | Metabolic biomarkers of Alzheimer's disease and mild cognitive impairment replicated in both case-control and nested case-control studies.

\begin{tabular}{lccc}
\hline Metabolites' Name & HMDB ID & \multicolumn{2}{c}{ Biosample's type } \\
\cline { 3 - 4 } & & AD vS. CN (reported frequency ${ }^{\text {a }}$ & CN_AD VS. CN (reported frequency) \\
\hline Palmitic acid & HMDB0000220 & Brain tissue, serum (6) & Serum (1) \\
Stearic acid & HMDB0000827 & Brain tissue, serum (2) & Serum (1) \\
Linoleic acid & HMDB0000673 & Brain tissue, serum (2) & Serum (1) \\
Glutamine & HMDB0000641 & Brain tissue, serum (4) (3) & Serum (1) \\
Oleic acid & HMDB0000207 & Serum (2) & Serum (1) \\
Myristic acid & HMDB0000806 & & \\
\hline
\end{tabular}

\begin{tabular}{lccc}
\hline & & & Biosample's type \\
\cline { 3 - 4 } & & AD VS. MCl (reported frequency) & MCl_AD VS. MCI (reported frequency) \\
\hline Arginine & HMDB0000517 & Plasma, CSF (1) & CSF (1) \\
Creatine & HMDB0000064 & Serum (2) & Plasma, CSF (2) \\
Histidine & HMDB0000177 & Serum (2) & CSF (1)
\end{tabular}

$A D$ VS. CN, AD VS. MCl, the comparison in case-control studies. CN_AD VS. CN, MCl_AD VS. MCl, the comparison in nested case-control studies. AD, Alzheimer's disease; $C N$, healthy controls; $\mathrm{MCl}$, mild cognitive impairment. ${ }^{a}$ Reported frequency of the metabolite in previous studies included in this systematic review. 


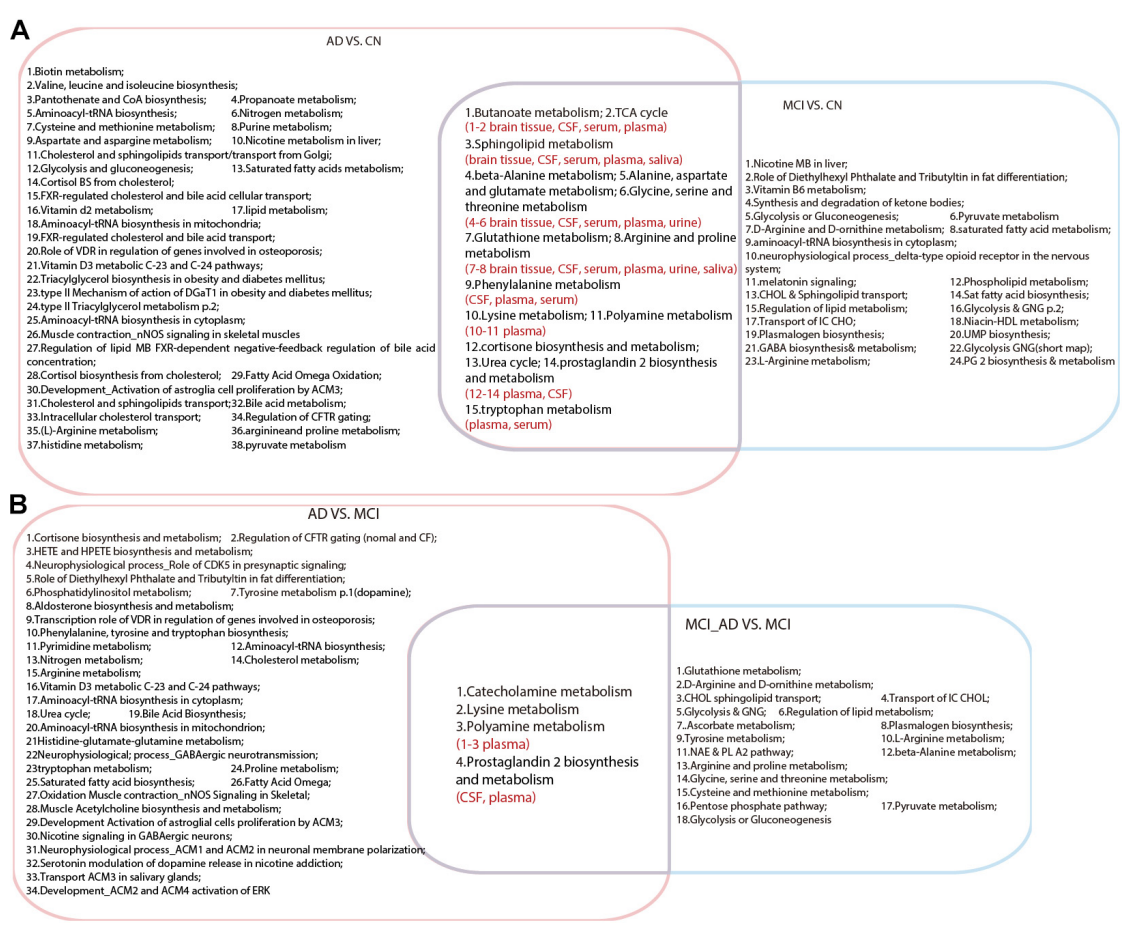

FIGURE 1 | Venn diagram illustrating shared and unique pathways in Alzheimer's disease and mild cognitive impairment in retrospective and prospective studies. (A) Common pathways between AD VS. CN and MCI VS. CN. (B) Common pathways between AD VS. MCl and MCI VS. MCl_AD (conversion from MCl to AD). AD, Alzheimer's disease; $\mathrm{CN}$, healthy controls; $\mathrm{MCl}$, mild cognitive impairment.

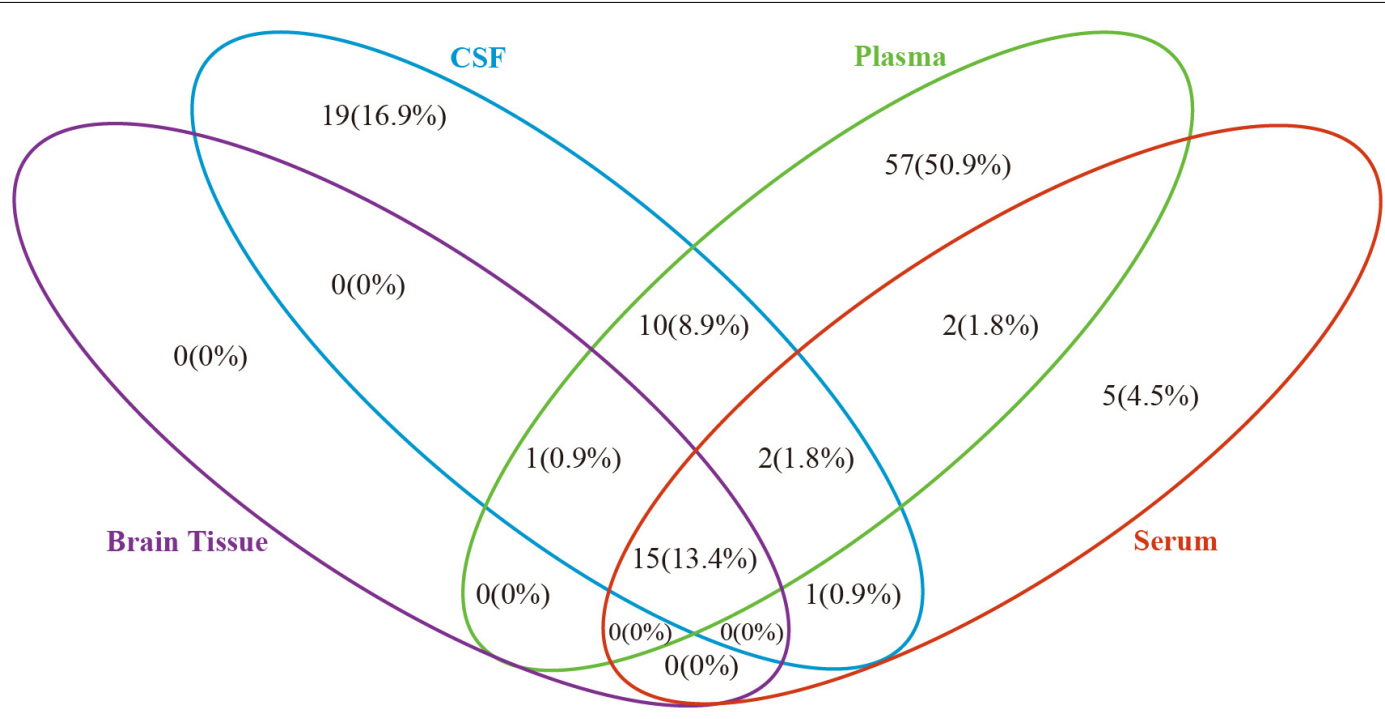

FIGURE 2 | Venn diagram depicting common and unique pathways altered in Alzheimer's disease in different biosample types. Blue oval represents cerebrospinal fluid, green oval represents plasma, purple oval represents brain tissue and red oval represents serum.

Pathway Intersection Analysis Among Different Types of Biosamples in AD

Based on the metabolites extracted from original studies, we performed pathway enrichment analyses by categorizing different types of samples (Supplementary Table 10). Supplementary Table 10 presents the 16, 48, 87, and 25 pathways of $\mathrm{AD}$ and $\mathrm{MCI}$ enriched in brain tissue, CSF, plasma, and serum, respectively. Figure 2 showed the comparison result of $\mathrm{AD}$, there were 15 common pathways perturbed in all four types of samples, such as sphingolipid metabolism, butanoate metabolism, propanoate metabolism, pantothenate and CoA biosynthesis, aminoacyl-tRNA biosynthesis and 


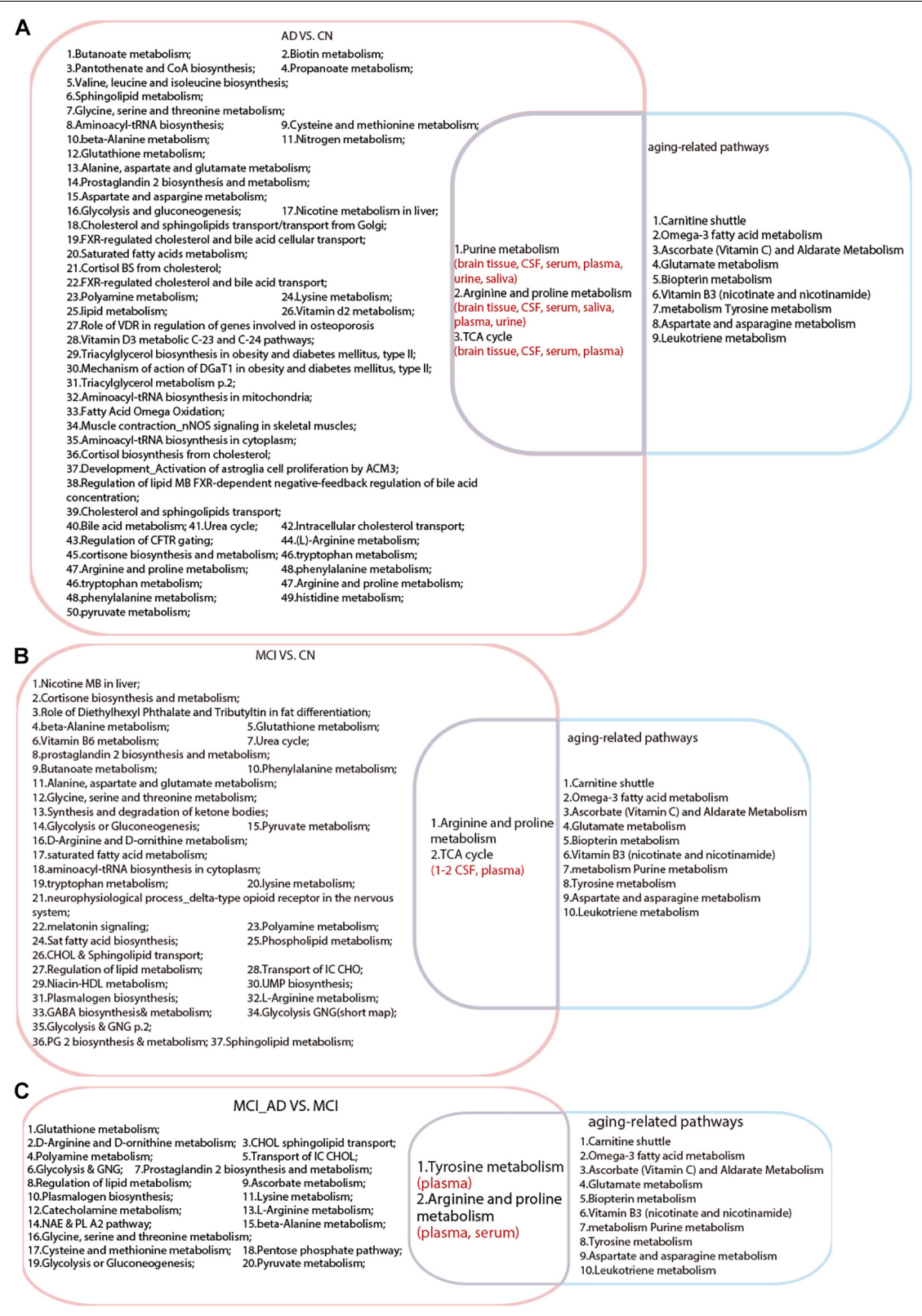

FIGURE 3 | Intersection analysis among pathways in Alzheimer's disease, mild cognitive impairment and aging. (A) Common pathways between AD VS. CN and aging. (B) Common pathways between $\mathrm{MCl}$ VS. CN and aging. (C) Common pathways between MCI VS. MCl_AD (conversion from MCl to AD) and aging. AD, Alzheimer's disease; $\mathrm{CN}$, healthy controls; $\mathrm{MCl}$, mild cognitive impairment.

some amino acid metabolism pathways. All 16 pathways in brain tissue and most pathways (28/48) in CSF could be detected in plasma.

\section{Intersection Analysis of Metabolic Pathways Among $\mathrm{AD}, \mathrm{MCl}$, and Aging}

We compared the aging-related pathways in the two-stage metabolomics analysis with the AD and MCI pathways generated in this systematic review. As shown in Figure 3, three pathways (including purine metabolism, arginine and proline metabolism, and the TCA cycle) revealed the common metabolic changes in
$\mathrm{AD}$ and aging and provided explanations for why natural aging is closely related to the high incidence of AD. All of these pathways have been detected in brain tissue, CSF, serum, and plasma in the $\mathrm{AD}$ population. Additionally, two pathways (arginine and proline metabolism and the TCA cycle) were found to be duplicated between MCI VS. CN and aging-related pathways. In particular, arginine and proline metabolism were duplicated between MCI_AD VS. MCI and aging-related pathways, which indicated that this mechanism plays an important role in the progression from healthy to $\mathrm{MCI}$ and to $\mathrm{AD}$ in the natural aging population. 


\section{Metabolic Biomarkers of AD in the Natural Aging Population}

Furthermore, we compared the top 30 metabolites of aging with the metabolites of $\mathrm{AD}$ and MCI reported in previous studies. After comparing Table 1 with Supplementary Tables 4, 5, 3 metabolites were identified that were related to both $\mathrm{AD}$ and aging, including 16-a-hydroxypregnenolone, stearic acid, and PC[16:0/22:5(4Z,7Z,10Z,13Z,16Z)], which can be considered metabolic biomarkers of $\mathrm{AD}$ in the natural aging population.

\section{DISCUSSION}

With aging, the probability of suffering from AD increases annually. Considering the dramatic aging of populations worldwide, it is of great importance to explore common mechanisms between $\mathrm{AD}$ and natural aging. The key finding of this study is the systematic comparison of the metabolic mechanisms of $\mathrm{AD}, \mathrm{MCI}$, and aging. We first revealed the metabolic mechanism and potential biomarkers of $\mathrm{AD}$ in a natural aging population.

Based on this independent metabolomics analysis in a twostage cross-sectional study, we found metabolites and pathways associated with aging. Strikingly, pathways enriched by agingrelated metabolites were consistent with the hypotheses of aging mechanisms in previous studies, such as oxidative stress (e.g., carnitine shuttle Yu et al., 2012), and inflammation (e.g., leukotriene metabolism). Additionally, in our work, pathways altered in the elderly were also related to various amino acid pathways. Previous detection of amino acids in the serum of normal healthy Japanese people revealed that the concentrations of aspartate, asparagine, and arginine increased with age in males, whereas the levels of tyrosine asparagine, arginine and proline increased with age in females, which together suggests that aspartate and asparagine metabolism and arginine and proline metabolism are related to aging (Kouchiwa et al., 2012). Furthermore, delayed degradation of plasma tyrosine (precursor of dopamine) in the elderly may influence cognition disruption during aging (Rudman et al., 1991; Berry et al., 2016). All of these metabolites also recurred in our study.

Previous studies have proven that oxidative stress plays a vital role in the progression of AD (Perry et al., 2002; Huang et al., 2016), as well as in MCI. The shared pathways between AD and MCI in our results reflected that MCI and $\mathrm{AD}$ have the same mechanisms. Glutathione, made up of cysteine, glycine and glutamate, was shown to have neuroprotective effects by reducing $\mathrm{A} \beta$-related oxidative stress via 4-hydroxynonenal (Mark et al., 1997) and attenuating amyloid fibrillation (Wang et al., 2009). Furthermore, lipid metabolism is one of the most extensively implicated dysfunctions in the context of AD. Consistent with that, cholesterol and sphingolipid transport, saturated fatty acid metabolism and sphingolipid metabolism were disrupted in both $\mathrm{AD}$ and $\mathrm{MCI}$. Moreover, disrupted amino acid pathways may be related to alterations of neurotransmitters. In our results, four pathways overlapped between AD VS. MCI and MCI_AD VS. MCI, including lysine metabolism, polyamine metabolism, catecholamine metabolism, and prostaglandin 2 biosynthesis and metabolism. Specific lysine residues within the microtubulebinding motif are the major sites of tau acetylation, which can inhibit tau function as a result of impaired tau-microtubule interactions and promote pathological tau aggregation (Cohen et al., 2011). Changes of polyamine metabolism in the brain influence the progression of $\mathrm{AD}$ through several mechanisms, such as the regulation of cholinergic neurotransmission (Mahajan et al., 2020). These pathway alterations indicated progressive changes in the patients from MCI to AD.

As shown in Figure 2, we compared the pathway results obtained in four biological samples from previous studies, and most of the pathway enrichment results are contained in plasma. Although there are some differences in the quantity of metabolites in different biological samples, most changes in the metabolites in the brain or CSF will be reflected in the plasma, in particular, all metabolic changes in the brain tissue of AD patients can be detected in plasma. Thus, plasma metabolites will most likely be the source of non-invasive markers. This provides us with the possibility of early screening of patients with MCI or $\mathrm{AD}$ in the aging population. From the mechanism point of view, perhaps $\mathrm{AD}$ related lesions in brain tissue may also be the result of accumulation or reduction of certain metabolites in plasma. We hope that our results can provide more inspirations for the study of relevant mechanisms. Moreover, we still cannot ignore the fact that more than $50 \%$ of metabolic changes in plasma cannot be evidenced in brain tissue and may be caused by other dietary or environmental exposures. Thus, the uniqueness of plasma AD markers should be given more attention in clinical studies.

As illustrated in Table 3, some fatty acids were found to be altered in both retrospective and prospective studies. In astroglia, palmitic acid may stimulate ceramide synthesis by secreting signaling molecules such as cytokines and nitric oxide, resulting in $\mathrm{A} \beta$ accumulation and tau hyperphosphorylation (Patil et al., 2007). Similarly, stearic, linoleic, and oleic acids were proven to be related to the accumulation of both $A \beta$ and tau in vitro (Patil and Chan, 2005; Amtul et al., 2012). Five metabolites (palmitic acid, stearic acid, linoleic acid, glutamine, and oleic acid) in serum/plasma have also been confirmed in brain tissue, which suggests their powerful potential for the non-invasive diagnosis of $\mathrm{AD}$. Moreover, arginine, creatine and histidine were observed in both retrospective and prospective studies. Considering that arginine and histidine were altered in both CSF and plasma/serum, these two metabolites may act as noninvasive biomarkers for the MCI population to monitor the progression from MCI to $\mathrm{AD}$.

The novel findings in our study are the metabolic pathways and biomarkers related to both aging and $\mathrm{AD}$. Regarding the three shared pathways between aging and AD, the TCA cycle did not attract our attention because it is such an extensive metabolic pathway altered in diverse physiological and pathological processes, which include the preclinical stage of AD (Atamna and Frey, 2007). Purine nucleoside phosphorylase (PNP) converts guanosine to guanine and inosine to hypoxanthine and is an important enzyme involved in purine metabolism. A study on astroglia reported a marked increase in PNP with aging (ZorefShani et al., 1995), while another study observed increased PNP activity in patients with AD (Alonso-Andres et al., 2018). 
Regarding arginine and proline metabolism, arginine is the central substance and serves as the only precursor of nitric oxide (NO). $\mathrm{NO}$ could react with superoxide $\left(\mathrm{O}_{2}-\right)$ to produce peroxynitrite $(\mathrm{ONOO}-)$, and the latter is so active that it would experience cleavage and generate reactive oxygen/nitrogen species (ROS/RNS) (Yi et al., 2009), which could occur in the process of natural aging. In addition, the brain is much more vulnerable to nitroxidative stress than other tissues due to its high oxygen demand, weakened antioxidative ability and low proliferative trait of neurons, indicating that oxidative stress is involved in the initiation of AD in healthy individuals (Kern and Behl, 2009). Arginine could be metabolized to agmatine, which is involved in memory decline processes and can be found in both elderly and AD brain tissue (Liu et al., 2014). Arginine and proline metabolism contains several metabolic pathways we mentioned in the AD-related pathways, such glutathione, glycine, and polyamine metabolism. Evidence has shown that these pathways are related to aging (Minois et al., 2011; Hashizume et al., 2015; Weschawalit et al., 2017). We can see that arginine and proline metabolism has been shown to play a role in prospective studies of the progression from no disease to $\mathrm{MCI}$ and eventually $\mathrm{AD}$; likewise, arginine and proline metabolism appears in the pathways related to natural aging.

From the results of direct comparison of metabolite lists of aging and $\mathrm{AD}$, there are three metabolites that were found to be duplicated: 16-a-hydroxypregnenolone, stearic acid and PC[16:0/22:5(4Z,7Z,10Z,13Z,16Z)]. 16-a-hydroxypregnenolone is classified as a gluco/mineralocorticoid, a progestogin or aprogestogin derivative. Although no cytological mechanism studies have confirmed the role of 16-a-hydroxypregnenolone in aging and dementia, we observed that it was significantly associated with aging in our metabolomics analysis and altered in AD patients in population studies (Vankova et al., 2016). Thus, experimental confirmation based on in vitro studies is urgently needed.

Strikingly, studies have demonstrated the role of stearic acid and PC[16:0/22:5(4Z,7Z,10Z,13Z,16Z)] in the pathological process of $\mathrm{AD}$. Together with a recent study demonstrating a close relationship between tau protein and inflammatory signaling in astrocytes (Lemoine et al., 2017), we assume a possible pathological process in astrocytes combining aging and $\mathrm{AD}$ via inflammatory and oxidative responses. Patil and Chan (2005) found that astroglia-mediated oxidative stress may be related to stearic and palmitic fatty acidinduced hyperphosphorylation of tau. Investigations have shown that in astrocytes, stearic acid promotes the release of inflammatory factors such as IL-6 and TNF $\alpha$ (Gupta et al., 2012). PC[16:0/22:5(4Z,7Z,10Z,13Z,16Z)] can be classified as PtdCho, which can be synthesized from cytidine diphosphate choline (CDP-choline) and diacylglycerol and contains long-chain polyunsaturated fatty acids, which are important components of neuron membranes. Wurtman (1992) proposed that choline was used to synthesize both acetylcholine (Ach) and PtdCho. Therefore, PtdCho could be taken to maintain the level of Ach when the body experiences a shortage of choline. Choline deficiency could occur in the contexts of both aging and $\mathrm{AD}$, resulting in depletion of PtdCho and death of cholinergic neurons. This "autocannibalism" hypothesis partially explained the selective vulnerability of the cholinergic system and provided clues regarding PtdCho as a shared metabolite of natural aging and $\mathrm{AD}$.

The limitations of our study are that we did not conduct in vitro experiments to verify the overlapping mechanisms between aging and AD. Although we used training and testing sets to screen out the aging-related metabolites that can be stably detected, our metabolomics research was a non-targeted test. It is necessary to further verify and analyze the sensitivity and specificity of metabolic markers based on targeted quantitative detection of a larger sample in a cohort population.

In conclusion, this study is the first to comprehensively compare metabolites and pathways between aging and AD by utilizing metabolomic measurement and systematic review. We proposed potential non-invasive biomarkers for $\mathrm{AD}$ diagnosis and MCI monitoring based on retrospective and prospective population studies. More importantly, we revealed the key role of arginine and proline metabolism in the progression from a healthy status to $\mathrm{MCI}$ to $\mathrm{AD}$ in a natural aging population. In particular, we provided potential metabolic markers (16-a-hydroxypregnenolone, stearic acid, and $\mathrm{PC}[16: 0 / 22: 5(4 Z, 7 Z, 10 Z, 13 Z, 16 Z)]$ of $\mathrm{AD}$ diagnosis for future validation in a natural aging population.

\section{DATA AVAILABILITY STATEMENT}

The datasets presented in this study can be found in online repositories. The names of the repository/repositories and accession number(s) can be found in the article/Supplementary Material.

\section{ETHICS STATEMENT}

The studies involving human participants were reviewed and approved by the Human Research and Ethics Committee of Harbin Medical University. The patients/participants provided their written informed consent to participate in this study.

\section{AUTHOR CONTRIBUTIONS}

FW and MW contributed to the study design, data interpretation, study supervision, and the acquisition of funding. YZ contributed to critical revision of the manuscript for important intellectual content. ZL and YY contributed to data processing, statistical analysis, and identification of differential metabolites. $\mathrm{CP}, \mathrm{KX}$, and YY contributed to questionnaire and sample collection. $\mathrm{ZW}$, VD, and CX contributed to sample preparation and metabolomics detection. ZL, YY, and LL contributed to literature search, quality assessment of individual studies and data extraction. KX and QQ contributed to manuscript preparation. All authors contributed to review and revision of the manuscript. 


\section{FUNDING}

This work was supported by grants from the National Natural Science Foundation of China (81773503 and 81973036), the Scientific Research Foundation for the Returned Overseas Scholars of Heilongjiang Province (LC2018033), the Capital Medical University (PYZ19137), the Capital's Funds for Health Improvement and Research (CFH 2020-4-1033), and the 2019 Harbin Medical University Graduate Research and Practice Innovation Project (YJSKYCX2019-76HYD).

\section{ACKNOWLEDGMENTS}

This manuscript has been released as a pre-print at Research Square (Xie et al., 2020).

\section{REFERENCES}

Alonso-Andres, P., Albasanz, J. L., Ferrer, I., and Martin, M. (2018). Purine-related metabolites and their converting enzymes are altered in frontal, parietal and temporal cortex at early stages of Alzheimer's disease pathology. Brain Pathol. 28, 933-946. doi: 10.1111/bpa.12592

Amtul, Z., Uhrig, M., Wang, L., Rozmahel, R. F., and Beyreuther, K. (2012). Detrimental effects of arachidonic acid and its metabolites in cellular and mouse models of Alzheimer's disease: structural insight. Neurobiol. Aging 33, 831.e21-31. doi: 10.1016/j.neurobiolaging.2011.07.014

Atamna, H., and Frey, W. H. II (2007). Mechanisms of mitochondrial dysfunction and energy deficiency in Alzheimer's disease. Mitochondrion 7, 297-310. doi: 10.1016/j.mito.2007.06.001

Bartus, R. T. (2000). On neurodegenerative diseases, models, and treatment strategies: lessons learned and lessons forgotten a generation following the cholinergic hypothesis. Exper. Neurol. 163, 495-529. doi: 10.1006/exnr.2000. 7397

Bender, A., Krishnan, K. J., Morris, C. M., Taylor, G. A., Reeve, A. K., Perry, R. H., et al. (2006). High levels of mitochondrial DNA deletions in substantia nigra neurons in aging and Parkinson disease. Nat. Genet. 38, 515-517. doi: $10.1038 / \mathrm{ng} 1769$

Berry, A. S., Shah, V. D., Baker, S. L., Vogel, J. W., O’Neil, J. P., Janabi, M., et al. (2016). Aging affects dopaminergic neural mechanisms of cognitive flexibility. J. Neurosci. 36, 12559-12569. doi: 10.1523/JNEUROSCI.0626-16.2016

Cohen, T. J., Guo, J. L., Hurtado, D. E., Kwong, L. K., Mills, I. P., Trojanowski, J. Q., et al. (2011). The acetylation of tau inhibits its function and promotes pathological tau aggregation. Nat. Commun. 2:252. doi: 10.1038/ncomms1255

Fleisher, A. S., Chen, K., Quiroz, Y. T., Jakimovich, L. J., Gomez, M. G., Langois, C. M., et al. (2012). Florbetapir PET analysis of amyloid- $\beta$ deposition in the presenilin 1 E280A autosomal dominant Alzheimer's disease kindred: a crosssectional study. Lancet Neurol. 11, 1057-1065. doi: 10.1016/s1474-4422(12) 70227-2

Greilberger, J., Koidl, C., Greilberger, M., Lamprecht, M., Schroecksnadel, K., Leblhuber, F., et al. (2008). Malondialdehyde, carbonyl proteins and albumindisulphide as useful oxidative markers in mild cognitive impairment and Alzheimer's disease. Free Rad. Res. 42, 633-638. doi: 10.1080/107157608 02255764

Gron, G., Brandenburg, I., Wunderlich, A. P., and Riepe, M. W. (2006). Inhibition of hippocampal function in mild cognitive impairment: targeting the cholinergic hypothesis. Neurobiol. Aging 27, 78-87. doi: 10.1016/j. neurobiolaging.2004.12.005

Gupta, S., Knight, A. G., Gupta, S., Keller, J. N., and Bruce-Keller, A. J. (2012). Saturated long-chain fatty acids activate inflammatory signaling in astrocytes. J. Neurochem. 120, 1060-1071. doi: 10.1111/j.1471-4159.2012.07660.x

Hashizume, O., Ohnishi, S., Mito, T., Shimizu, A., Ishikawa, K., Nakada, K., et al. (2015). Epigenetic regulation of the nuclear-coded GCAT and SHMT2

\section{SUPPLEMENTARY MATERIAL}

The Supplementary Material for this article can be found online at: https://www.frontiersin.org/articles/10.3389/fcell.2021. 602887/full\#supplementary-material

Supplementary Figure 1 | Variable importance for the top 30 aging-related metabolites selected by random forest. The $y$-axis represents retention time and mass of metabolites. The $x$-axis represents IncNodePurity, which indicates the importance of the variable given by the classification model.

Supplementary Figure 2 | Flow diagram of the literature search of metabolomics studies in Alzheimer's disease using the PubMed, Cochrane and Embase databases.

Supplementary Figure 3 | Description of the included studies' basic information. (A) year of publication; (B) race of population; (C) study design; (D) different platforms used in different biosample analyses.

genes confers human age-associated mitochondrial respiration defects. Sci. Rep. 5:10434. doi: 10.1038/srep14591

Hertel, J., Friedrich, N., Wittfeld, K., Pietzner, M., Budde, K., Van der Auwera, S., et al. (2016). Measuring biological age via metabonomics: the metabolic age score. J. Proteom. Res. 15, 400-410. doi: 10.1021/acs.jproteome.5b00561

Huang, W. J., Zhang, X., and Chen, W. W. (2016). Role of oxidative stress in Alzheimer's disease. Biomed. Rep. 4, 519-522. doi: 10.3892/br.2016.630

Jack, C. R., Knopman, D. S., Jagust, W. J., Shaw, L. M., Aisen, P. S., Weiner, M. W., et al. (2010). Hypothetical model of dynamic biomarkers of the Alzheimer's pathological cascade. Lancet Neurol. 9:119. doi: 10.1016/s1474-4422(09) 70299-6

Kern, A., and Behl, C. (2009). The unsolved relationship of brain aging and lateonset Alzheimer disease. Biochim. Biophys. Acta 1790, 1124-1132. doi: 10.1016/ j.bbagen.2009.07.016

Kouchiwa, T., Wada, K., Uchiyama, M., Kasezawa, N., Niisato, M., Murakami, H., et al. (2012). Age-related changes in serum amino acids concentrations in healthy individuals. Clin. Chem. Lab. Med. 50, 861-870. doi: 10.1515/cclm2011-0846

Lane, C. A., Hardy, J., and Schott, J. M. (2017). Alzheimer's disease. Eur. J. Neurol. 25, 59-70. doi: 10.1111/ene.13439

Lemoine, L., Saint-Aubert, L., Nennesmo, I., Gillberg, P. G., and Nordberg, A. (2017). Cortical laminar tau deposits and activated astrocytes in Alzheimer's disease visualised by (3)H-THK5117 and (3)H-deprenyl autoradiography. Sci. Rep. 7:45496. doi: 10.1038/srep45496

Liu, P., Fleete, M. S., Jing, Y., Collie, N. D., Curtis, M. A., Waldvogel, H. J., et al. (2014). Altered arginine metabolism in Alzheimer's disease brains. Neurobiol. Aging 35, 1992-2003. doi: 10.1016/j.neurobiolaging.2014.03.013

Lumbreras, B., Porta, M., Marquez, S., Pollan, M., Parker, L. A., and HernandezAguado, I. (2008). QUADOMICS: an adaptation of the Quality Assessment of Diagnostic Accuracy Assessment (QUADAS) for the evaluation of the methodological quality of studies on the diagnostic accuracy of '-omics'-based technologies. Clin. Biochem. 41, 1316-1325. doi: 10.1016/j.clinbiochem.2008. 06.018

Mahajan, U. V., Varma, V. R., Griswold, M. E., Blackshear, C. T., An, Y., Oommen, A. M., et al. (2020). Dysregulation of multiple metabolic networks related to brain transmethylation and polyamine pathways in Alzheimer disease: a targeted metabolomic and transcriptomic study. PLoS Med. 17:e1003012. doi: 10.1371/journal.pmed.1003012

Mark, R. J., Lovell, M. A., Markesbery, W. R., Uchida, K., and Mattson, M. P. (1997). A role for 4-hydroxynonenal, an aldehydic product of lipid peroxidation, in disruption of ion homeostasis and neuronal death induced by amyloid beta-peptide. J. Neurochem. 68, 255-264. doi: 10.1046/j.1471-4159.1997.6801 0255.x

Markesbery, W. R., and Lovell, M. A. (2007). Damage to lipids, proteins, DNA, and RNA in mild cognitive impairment. Archiv. Neurol. 64, 954-956. doi: 10.1001/archneur.64.7.954 
Mayeux, R., and Stern, Y. (2012). Epidemiology of alzheimer disease. Cold Spring Harb. Perspect. Med. 2:a006239. doi: 10.1101/cshperspect.a006239

Mielke, M. M., and Lyketsos, C. G. (2006). Lipids and the pathogenesis of Alzheimer's disease: is there a link? Intern. Rev. Psychiatry 18, 173-186. doi: $10.1080 / 09540260600583007$

Minois, N., Carmona-Gutierrez, D., and Madeo, F. (2011). Polyamines in aging and disease. Aging 3, 716-732. doi: 10.18632/aging.100361

Naslund, J., Haroutunian, V., Mohs, R., Davis, K. L., Davies, P., Greengard, P., et al. (2000). Correlation between elevated levels of amyloid beta-peptide in the brain and cognitive decline. JAMA 283, 1571-1577. doi: 10.1001/jama.283.12. 1571

Nichols, E., Szoeke, C. E. I., Vollset, S. E., Abbasi, N., Abd-Allah, F., Abdela, J., et al. (2019). Global, regional, and national burden of Alzheimer's disease and other dementias, 1990-2016: a systematic analysis for the Global Burden of Disease Study 2016. Lancet Neurol. 18, 88-106. doi: 10.1016/s1474-4422(18)30403-4

Nicholson, J. K., Holmes, E., and Elliott, P. (2008). The metabolome-wide association study: a new look at human disease risk factors. J. Proteom. Res. 7, 3637-3638. doi: 10.1021/pr8005099

Ohm, T. G., Muller, H., Braak, H., and Bohl, J. (1995). Close-meshed prevalence rates of different stages as a tool to uncover the rate of Alzheimer's diseaserelated neurofibrillary changes. Neuroscience 64, 209-217. doi: 10.1016/03064522(95)90397-P

Patil, S., and Chan, C. (2005). Palmitic and stearic fatty acids induce Alzheimerlike hyperphosphorylation of tau in primary rat cortical neurons. Neurosci. Lett. 384, 288-293. doi: 10.1016/j.neulet.2005.05.003

Patil, S., Melrose, J., and Chan, C. (2007). Involvement of astroglial ceramide in palmitic acid-induced Alzheimer-like changes in primary neurons. Eur. J. Neurosci. 26, 2131-2141. doi: 10.1111/j.1460-9568.2007.05797.x

Perry, G., Cash, A. D., and Smith, M. A. (2002). Alzheimer disease and oxidative stress. J. Biomed. Biotechnol. 2, 120-123. doi: 10.1155/S1110724302203010

Poon, H. F., Shepherd, H. M., Reed, T. T., Calabrese, V., Stella, A. M., Pennisi, G., et al. (2006). Proteomics analysis provides insight into caloric restriction mediated oxidation and expression of brain proteins associated with agerelated impaired cellular processes: mitochondrial dysfunction, glutamate dysregulation and impaired protein synthesis. Neurobiol. Aging 27, 1020-1034. doi: 10.1016/j.neurobiolaging.2005.05.014

Psychogios, N., Hau, D. D., Peng, J., Guo, A. C., Mandal, R., Bouatra, S., et al. (2011). The human serum metabolome. PLoS One 6:e16957. doi: 10.1371/ journal.pone.0016957

Rist, M. J., Roth, A., Frommherz, L., Weinert, C. H., Kruger, R., Merz, B., et al. (2017). Metabolite patterns predicting sex and age in participants of the Karlsruhe Metabolomics and Nutrition (KarMeN) study. PLoS One 12:e0183228. doi: 10.1371/journal.pone.0183228

Rudman, D., Abbasi, A. A. L., Chaudry, F., and Mattson, D. E. (1991). Delayed plasma clearance of phenylalanine and tyrosine in elderly men. J. Am. Geriatr. Soc. 39, 33-38. doi: 10.1111/j.1532-5415.1991.tb05903.x

Vankova, M., Hill, M., Velikova, M., Vcelak, J., Vacinova, G., Dvorakova, K., et al. (2016). Preliminary evidence of altered steroidogenesis in women with
Alzheimer's disease: have the patients "OLDER" adrenal zona reticularis? J. Steroid Biochem. Mol. Biol. 158, 157-177. doi: 10.1016/j.jsbmb.2015.12.011

Wang, J., Markesbery, W. R., and Lovell, M. A. (2006). Increased oxidative damage in nuclear and mitochondrial DNA in mild cognitive impairment. J. Neurochem. 96, 825-832. doi: 10.1111/j.1471-4159.2005.03615.x

Wang, S. S., Chou, S. W., Liu, K. N., and Wu, C. H. (2009). Effects of glutathione on amyloid fibrillation of hen egg-white lysozyme. Intern. J. Biol. Macromol. 45, 321-329. doi: 10.1016/j.ijbiomac.2009.08.003

Weschawalit, S., Thongthip, S., Phutrakool, P., and Asawanonda, P. (2017). Glutathione and its antiaging and antimelanogenic effects. Clin. Cosmet. Invest. Dermatol. 10, 147-153. doi: 10.2147/CCID.S128339

Whiting, P. F., Rutjes, A. W., Westwood, M. E., Mallett, S., Deeks, J. J., Reitsma, J. B., et al. (2011). QUADAS-2: a revised tool for the quality assessment of diagnostic accuracy studies. Ann. Intern. Med. 155, 529-536. doi: 10.7326/ 0003-4819-155-8-201110180-00009

Wilkins, J. M., and Trushina, E. (2017). Application of metabolomics in Alzheimer's disease. Front. Neurol. 8:719. doi: 10.3389/fneur.2017.00719

Wurtman, R. J. (1992). Choline metabolism as a basis for the selective vulnerability of cholinergic neurons. Trends Neurosci. 15, 117-122. doi: 10.1016/01662236(92)90351-8

Xie, K., Qin, Q., Long, Z., Yang, Y., Peng, C., Xi, C., et al. (2020). High-throughput metabolomics for discovering potential biomarkers and identifying metabolic mechanisms in aging and Alzheimer's disease. Research Square [Preprint]. doi: 10.21203/rs.3.rs-60073/v1

Yi, J., Horky, L. L., Friedlich, A. L., Shi, Y., Rogers, J. T., and Huang, X. (2009). L-arginine and Alzheimer's disease. Intern. J. Clin. Exper. Pathol. 2, 211-238.

Yu, Z., Zhai, G., Singmann, P., He, Y., Xu, T., Prehn, C., et al. (2012). Human serum metabolic profiles are age dependent. Aging Cell 11, 960-967. doi: 10.1111/j.1474-9726.2012.00865.x

Zhu, Y., Carvey, P. M., and Ling, Z. (2006). Age-related changes in glutathione and glutathione-related enzymes in rat brain. Brain Res. 1090, 35-44. doi: 10.1016/j.brainres.2006.03.063

Zoref-Shani, E., Bromberg, Y., Lilling, G., Gozes, I., Brosh, S., Sidi, Y., et al. (1995). Developmental changes in purine nucleotide metabolism in cultured rat astroglia. Intern. J. Dev. Neurosci. 13, 887-896. doi: 10.1016/0736-5748(95) 00054-2

Conflict of Interest: The authors declare that the research was conducted in the absence of any commercial or financial relationships that could be construed as a potential conflict of interest.

Copyright ( 2021 Xie, Qin, Long, Yang, Peng, Xi, Li, Wu, Daria, Zhao, Wang and Wang. This is an open-access article distributed under the terms of the Creative Commons Attribution License (CC BY). The use, distribution or reproduction in other forums is permitted, provided the original author(s) and the copyright owner(s) are credited and that the original publication in this journal is cited, in accordance with accepted academic practice. No use, distribution or reproduction is permitted which does not comply with these terms. 